\title{
Jamming in Systems Composed of Frictionless Ellipse-Shaped Particles
}

\author{
Mitch Mailman ${ }^{1}$, Carl F. Schreck ${ }^{2}$, Corey S. O’Hern² and Bulbul Chakraborty ${ }^{1}$ \\ ${ }^{1}$ Martin Fisher School of Physics, Brandeis University, Mail Stop 057, Waltham, MA 02454-9110, USA \\ ${ }^{2}$ Department of Mechanical Engineering, Yale University, New Haven, CT \\ 06520-8284 and Department of Physics, Yale University, New Haven CT 06520-8120 \\ (Dated: October 26, 2018)
}

\begin{abstract}
We study the structural and mechanical properties of jammed ellipse packings, and find that the nature of the jamming transition in these systems is fundamentally different from that for spherical particles. Ellipse packings are generically hypostatic with more degrees of freedom than constraints. The spectra of low energy excitations possess two gaps and three distinct branches over a range of aspect ratios. In the zero compression limit, the energy of the modes in the lowest branch increases quartically with deformation amplitude, and the density of states possesses a $\delta$-function at zero frequency. We identify scaling relations that collapse the low-frequency part of the spectra for different aspect ratios. Finally, we find that the degree of hypostaticity is determined by the number of quartic modes of the packing.
\end{abstract}

PACS numbers: 61.43.-j, 81.05.Kf, 63.50.Lm, 83.80.Fg

A decade ago, Liu and Nagel proposed that jamming transitions in glassy, granular, and other nonequilibrium amorphous systems can be described by the jamming phase diagram [1], and that a 'fixed point' (Point J) in the jamming phase diagram controls slow dynamics in these systems even far from Point J [2]. For model disordered systems composed of frictionless, spherical grains interacting via purely repulsive, shortrange potentials, the packings at Point $\mathrm{J}$ are isostatic [3, 4], where the number of degrees of freedom exactly matches the number of constraints. It has been shown that isostatic packings of spherical grains have interesting mechanical properties; for example, they possess an abundance of spatially extended "floppy modes" of excitation [5, 6, 7] and non-elastic response to applied deformations [8].

However, there have been relatively few theoretical studies of jamming in systems with aspherical particles, despite the fact that most physical particulate media have grains with anisotropic shapes. The introduction of aspherical particles in equilibrium systems gives rise to completely new phases of matter and critical behavior as evidenced in the field of liquid crystals. What is the nature of jamming transitions in nonequilibrium systems when the grains are aspherical? For example, is there still a special point $\mathbf{J}$ in the jamming phase diagram, where packings are isostatic, that controls slow dynamics? In this letter, we begin to address these important open questions by investigating the structural and mechanical properties of static packings of frictionless anisotropic particles.

For a static packing of $N$ grains in $d$ spatial dimensions, with $d-1$ rotational and $d$ translational degrees of freedom per grain, total force and torque balance on each grain can be satisfied only if the total number of contacts satisfies $N_{c} \geq N_{I} \equiv N(2 d-1)$ in the large system limit. Isostatic packings satisfy $N_{c}=N_{I}$, while hypostatic packings possess $N_{c}<N_{I}$. In contrast to spherical particle packings, static packings of ellipsoidal particles, studied previously by Donev, et al. [9], are generically hypostatic and possess higher densities without translational and orientational order. Experiments on packings of ellipsoidal $M \& M$ candies have also verified these results [10]. These previous studies raise several fundamental questions about static packings of ellipsoidal particles; for example, why are they hypostatic and what is the nature of their low-energy excitations? In this letter, we study the low-energy, vibrational excitations of 2D static packings of ellipses using a numerical packing-generation algorithm in which soft ellipses interact via purely repulsive potentials at zero temperature. Our analysis demonstrates the existence of two gaps in the vibrational spectrum over a range of aspect ratios. The energy of the modes below the first gap increases quartically with deformation amplitude along the soft directions, and the number of these quartic modes determines the degree of hypostaticity of the packings.

Compression packing-generation protocol We generated an extensive set of static packings of ellipses over a range of system sizes from $N=120$ to 1920 , in which particles are 'just touching', using a numerical packing-generation protocol similar to that employed to create static packings of spherical particles [11, 12]. We will refer to this protocol as the 'compression method'. In this method, soft, purely repulsive ellipses are first randomly deposited in a square cell with periodic boundary conditions at a low packing fraction $(\phi=0.5)$. The configurations are successively compressed in small steps $\left(\Delta \phi=10^{-4}\right)$ and then relaxed using conjugate energy minimization after each step. Near the jamming packing fraction $\phi_{J}$, where the particles are just touching, the configurations are expanded or compressed by decreasing amounts until the system has vanishingly small total potential energy per particle $V_{\text {tol }}<V<2 V_{\text {tol }} . V_{\text {tol }}=10^{-12}$ for most simulations, $V=\sum_{i>j} V\left(r_{i j}\right)$ summed over all ellipse pairs,

$$
V\left(r_{i j}\right)= \begin{cases}\left(1-r_{i j} / \sigma_{i j}\right)^{2} & r_{i j} \leq \sigma_{i j} \\ 0 & r_{i j}>\sigma_{i j}\end{cases}
$$

and $r_{i j}$ is the separation between the centers of mass of ellipses $i$ and $j$. The separations and orientations $\hat{u}_{i}$ and $\hat{u}_{j}$ of the long axes of ellipses $i$ and $j$ determine the Perram and Wertheim 


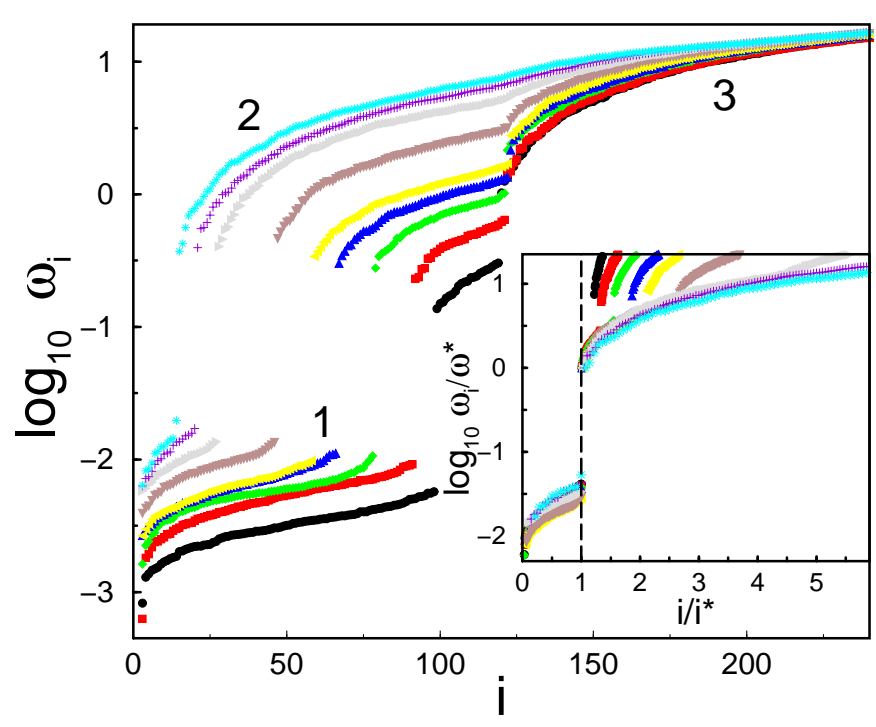

FIG. 1: (Color online) Normal mode frequencies $\omega_{i}$ from the dynamical matrix (Eq. 3) vs. index $i$, sorted by increasing frequency for $N=120$ ellipse packings at nine aspect ratios, $\alpha=1.02$ (black), 1.04 (red), 1.06 (green), 1.08 (blue), 1.1 (yellow), 1.2 (brown), 1.4 (gray), 1.6 (violet), and 1.80 (turquoise). The sorted frequency spectrum possesses three distinct branches (numbered 1, 2, and 3 ). In the inset, we show the scaled frequency $\omega_{i} / \omega^{*}$ vs. $i / i^{*}$, which collapses the low frequency part of the spectra at 1 (vertical dashed line).

overlap parameter [13, 14, 15, 16]

$$
\sigma_{i j}=\min _{\lambda} \frac{\sigma_{0}(\lambda)}{\sqrt{1-\frac{\chi(\lambda)}{2} \sum_{ \pm} \frac{\beta(\lambda) \hat{r}_{i j} \cdot \hat{u}_{i} \pm \beta^{-1}(\lambda) \hat{r}_{i j} \cdot \hat{u}_{j}}{1 \pm \chi(\lambda) \hat{u}_{i} \cdot \hat{u}_{j}}}},
$$

where $\sigma_{0}, \beta$, and $\chi$ depend on $\lambda$ and the major (minor) axis, $a_{i}\left(b_{i}\right)$, of the $i$ th ellipse [17]. To determine $\sigma_{i j}$ for each pair, minimization of the parameter $0<\lambda<1$ must be performed. We simulate bidisperse mixtures to inhibit translational and orientational order: one-third of the particles are large with the major axis 1.4 times that of the small particles [9]. Using this procedure, we generated an ensemble of at least 100 ellipse packings, each characterized by the jamming packing fraction $\phi_{J}$, over a range of aspect ratios from $\alpha=1$ to 2 . We calculated the global bond-orientational and nematic order parameters [18], and found no significant ordering with order parameters $\sim 1 / \sqrt{N}$ for all $\alpha$.

Vibrational Spectra To determine mechanical properties of ellipse packings, we calculate the dynamical matrix

$$
M_{m n}=\frac{\partial^{2} V}{\partial \xi_{m} \partial \xi_{n}}
$$

where $\xi_{m}=\left\{x_{m}, y_{m}, a_{m} \theta_{m}\right\}, x_{m}$ and $y_{m}$ are center of mass coordinates of ellipse $m, \theta_{m}$ is the angle between $\hat{u}_{m}$ and the $x$-axis, and $m, n=1, \cdots, N$ [19]. When Eq. 3 is evaluated for an ellipse packing and diagonalized using periodic boundary conditions, in principle one obtains $(2 d-1) N^{\prime}-d$ nontrivial vibrational eigenmodes, where $N^{\prime}=N-N_{r}$ and $N_{r}$ is the number of 'rattlers' with fewer than $d+1$ contacts. If we assume that all ellipses have the same mass,

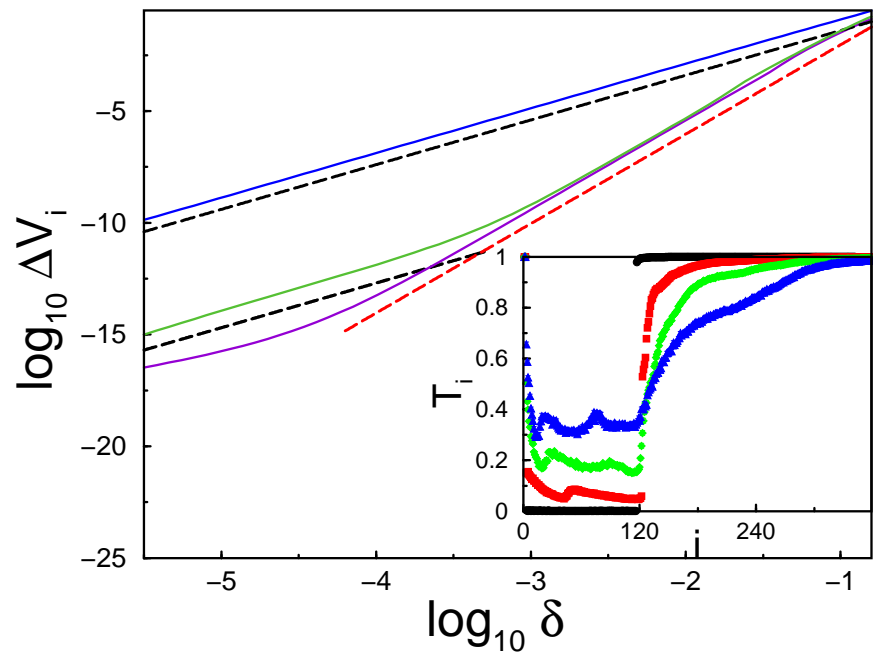

FIG. 2: (Color online) Change in potential energy $\Delta V_{i}$ vs. displacement $\delta$ along $\hat{e}_{i}$ for $N=120$ and $\alpha=1.5\left(i^{*}=22\right) . \Delta V_{i}$ (solid blue) for $i=115>i^{*}$ is quadratic in $\delta$. In contrast, for $i \leq i^{*}, \Delta V_{i} \propto \delta^{2}$ for $\delta<\delta_{c}$, but $\propto \delta^{4}$ for $\delta>\delta_{c}$. For $i=24$, we show that $\delta_{c}$ decreases

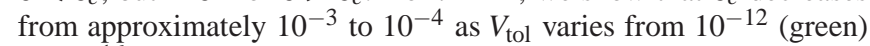
to $10^{-16}$ (purple). The dashed black (red) line has slope two (four). Inset: The translational contribution $T_{i}$ to the sum of the squares of the amplitudes of each eigenvector $\hat{e}_{i}$ of the dynamical matrix for aspect ratio $\alpha=1.01$ (black), 1.20 (red), 1.50 (green), and 2.00 (blue).

the square roots of the eigenvalues of the dynamical matrix give the normal mode frequencies $\omega_{i}$ indexed by $i$. We denote the normalized eigenvector corresponding to $\omega_{i}$ by $\hat{e}_{i}=\left\{e_{x i}^{j=1}, e_{y i}^{j=1}, e_{\theta i}^{j=1}, \cdots, e_{x i}^{j=N^{\prime}}, e_{y i}^{j=N^{\prime}}, e_{\theta i}^{j=N^{\prime}}\right\}$ with the constraint that $\hat{e}_{i}^{2}=1$. Below, we show the relative contributions of translational and orientational components of the eigenvectors, for example, the translational contribution from mode $i$ is $T_{i}=\sum_{j=1}^{N^{\prime}}\left\{\left(e_{x i}^{j}\right)^{2}+\left(e_{y i}^{j}\right)^{2}\right\}$ and $R_{i}=1-T_{i}$.

Over a range of aspect ratios, the spectrum $\omega_{i}$, sorted in order of increasing frequency, possesses three distinct regimes (cf Fig. 1): (1) modes with indexes $i-2<i^{*}(\alpha)$ below the low-frequency gap, (2) modes with index $i^{*} \leq i-2 \leq i_{t}=$ $(d-1) N$, where for $\alpha \leq \alpha_{t}$, there is a second gap at index $i_{t}$, and (3) modes with index $i-2>i_{t}$. (We are explicitly not including the two trivial modes corresponding to translational invariance.) In the inset to Fig. 11 we show that we are able to choose aspect ratio dependent scaling factors $\omega^{*}$ and $i^{*}$ that collapse the low-frequency part of the spectra including the first gap. We find that $\omega^{*}$ is roughly linear with $\alpha-1$, while $i^{*}$ possesses two different scaling regimes for $\alpha-1 \ll 1$ and $\alpha>1$. As demonstrated in the inset to Fig. 2] modes in regions (1) and (2) are mainly rotational, whereas those in region (3) correspond to mainly translational motion.

We find that our ellipse packings at finite, but small overcompression possess $(2 d-1) N-d$ nonzero, positive eigenvalues [20]. To rationalize this result with the fact that hard ellipse packings are generically hypostatic [9], we perturbed our ellipse packings along each of the eigendirections of the dynamical matrix over a range of overcompression. If $\vec{\xi}_{0}$ characterizes the centers of mass and orientations of the original 


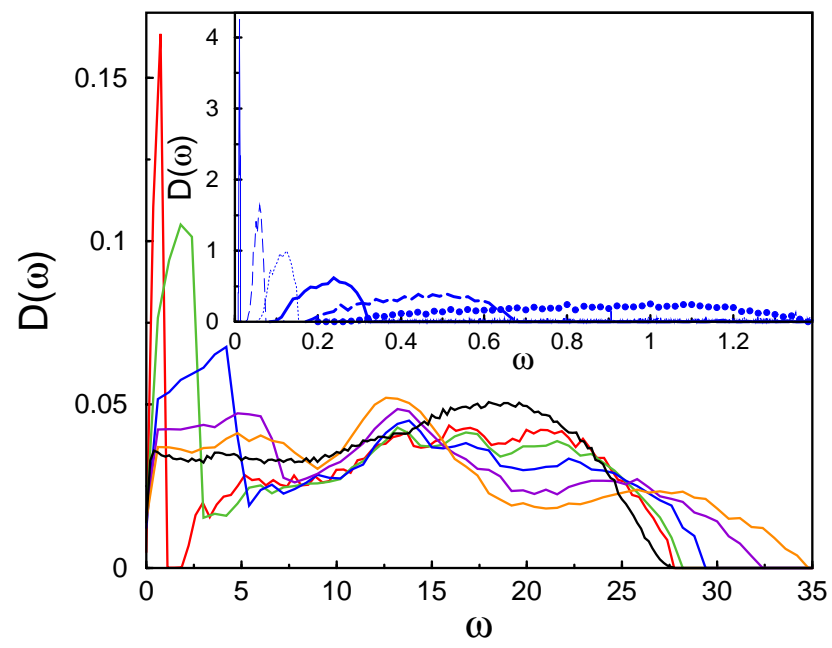

FIG. 3: Density of vibrational modes $D(\omega)$ for ellipse packings with $N=120$ for $\alpha=1.0$ (black), 1.1 (red), 1.2 (green), 1.4 (blue), 1.7 (violet), and 2.0 (orange). The low $\omega$ peak corresponds to rotational modes in frequency regime 2 discussed in the text. The inset magnifies the low $\omega$ region for $\alpha$ near 1: $\alpha=1.001$ (thin solid), 1.005 (thin dashed), 1.01 (thin dotted), 1.02 (thick solid), 1.04 (thick dashed), and 1.08 (filled circles). The low-frequency peak is sharp and $D(\omega)$ possesses a gap in $\omega$ for $\alpha<\alpha_{t} \approx 1.2$. However, the peak broadens and connects to $D(\omega)$ at large $\omega$ without going to zero for $\alpha>\alpha_{t}$.

static ellipse packing, the perturbed configuration obtained after a shift by $\delta$ along eigenmode $i$ and relaxation to the nearest local energy minimum is $\vec{\xi}_{i}=\vec{\xi}_{0}+\delta \hat{e}_{i}$. In Fig. 2, we plot the change in potential energy, $\Delta V_{i} \equiv V\left(\vec{\xi}_{i}\right)-V\left(\vec{\xi}_{0}\right)$, of ellipse packings with $N=120$ at $\alpha=1.5$ arising from a perturbation along mode $i$ as a function of amplitude $\delta$ and for two values of overcompression $V_{\text {tol }}$. As shown in Fig. 2, for modes with indexes in regions (2) and (3) of the frequency spectrum $\Delta V_{i} \propto \delta^{2}$ for all $\delta$ independent of $V_{\text {tol }}$. In contrast, there is range $\delta>\delta_{c}$ over which modes in region (1) display quartic dependence on $\delta, \Delta V_{i} \propto \delta^{4}$, whereas $\Delta V_{i} \propto \delta^{2}$ for $\delta<\delta_{c}$. Since $\delta_{c} \sim V_{\text {tol }}^{1 / 4}$ for modes in region (1), quartic behavior will persist over the entire range of $\delta$ in the zero compression limit. Thus, 'just-touching' ellipse packings are stabilized by quartic rather than quadratic terms in the expansion of the total potential energy around the reference packing[9].

The density of vibrational modes, $D(\omega)$, obtained from the spectrum, $\omega_{i}$, shown in Fig. 3, exhibits several key differences from that for disk packings [2]. First, the plateau at small $\omega$ characteristic of nearly-isostatic disk packings is replaced by a narrow peak at low frequencies. This peak is composed of modes in region (2) of the spectrum that display collective, primarily rotational motions. For $\alpha<\alpha_{t}$ (with $\alpha_{t} \approx 1.2$ for $N=120$ ), this peak is clearly separated by a gap from the broad, high-frequency regime. Note that on a logarithmic scale one would also see a peak that corresponds to modes in region (1), and is separated by a gap from the modes in region (2). As discussed before, this peak shifts to lower $\omega$, and narrows to a $\delta$-function at zero frequency in the zero-compression limit. Second, for large aspect ratios $(\alpha>1.5$ for $N=120)$, $D(\omega)$ has significantly more structure than that for disk pack-

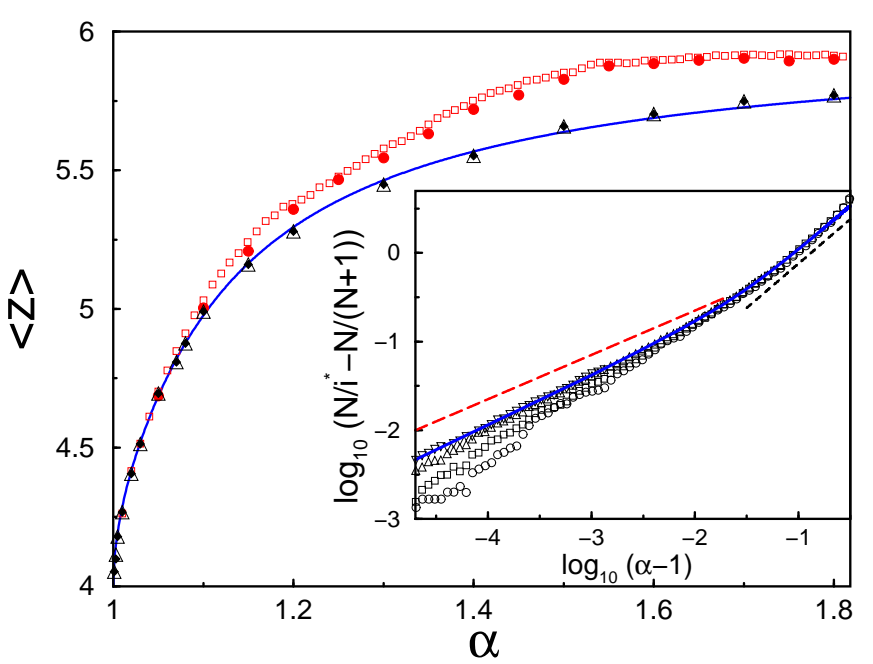

FIG. 4: (Color online) Contact number $\langle z\rangle$ vs. $\alpha$ for compression (triangles) and annealing $(\Delta \alpha=0.005$ (open squares) and 0.05 (filled circles)) methods for $N=480$. $\langle z\rangle$ for annealing diverges from that for compression for $\alpha>1.1$. The filled diamonds represent $\langle z\rangle=$ $6-i^{*}(\alpha) /(3 N)$ for the compression method data. The inset shows the system size dependence of $N / i^{*}(\alpha)-N /(N+1)$ for $N=120$ (circles), 240 (squares), 960 (upward triangles), and 1920 (downward triangles) for the compression data. The red (black) dashed line has slope 0.5 (1.0). The solid line is an interpolation between power-laws of 0.5 at small $\alpha-1$ and 1.0 at large $\alpha-1$, which was used to fit $\langle z\rangle$ in the main plot (solid blue line).

ings for $\omega>10$. These new features will be investigated in more detail in future work [21]. We have verified that the vibrational properties described here also hold for packings in which ellipses interact via the Gay-Berne potential [22] with a simpler form for the overlap function than in Eq.2.

Hypostaticity Our analysis of the structural and mechanical properties of ellipse packings has led to several novel observations: (1) The quartic modes that exist for hard ellipse packings develop a quadratic contribution at finite overcompression, and therefore compression stabilizes ellipse packings [9]; (2) The contribution of these quartic modes to the density of vibrational modes decreases as the aspect ratio increases; and (3) Ellipse packings are generically hypostatic, as shown in Fig.4, with smaller average contact number $\langle z\rangle$ than that predicted from isostatic counting arguments, i.e. $\langle z\rangle_{\text {iso }}=6$ for ellipses with $\alpha>1$. Hypostatic packings have fewer contacts than required to satisfy all force and torque balance conditions, and thus in some directions of configuration space these packings are only quartically, not quadratically stable. We find that quartic modes represent collective, primarily rotational motions of the ellipses, which do not lead to cage breaking and particle rearrangements. Thus, we expect that if the isostatic counting argument is reformulated so that the quartic modes are not constrained, $\langle z\rangle$ will correspond to the minimum number of contacts necessary to constrain the quadratic modes. Thus, ellipse packings are isostatic with respect to only the quadratic modes.

When each internal degree of freedom in an ellipse packing is stabilized, the isostatic conjecture gives $N\langle z\rangle / 2=3 N-1$. If 
it is not necessary to constrain the quartic modes, this equation can be rewritten as

$$
\frac{N\langle z\rangle}{2}=3 N-i^{*}(\alpha)
$$

where $N_{4}=i^{*}(\alpha)-1$ is the number of quartic modes in region 1 of the frequency spectrum. Thus, by measuring the number of quartic modes, we are able to determine $\langle z\rangle(\alpha)$ as shown in Fig. 4. In the inset we show that $N / i^{*}(\alpha)-N /(N+1)$ has two power-law regimes: scaling as $\sqrt{\alpha-1}(\alpha-1)$ for small (large) $\alpha-1$. In these limits, we obtain

$$
\langle z\rangle(\alpha)=\langle z\rangle(1)+2 \frac{A_{n}(\alpha-1)^{n}}{1+A_{n}(\alpha-1)^{n}},
$$

where $n=0.5$ (1) for small (large) $\alpha-1$ and $A_{0.5}$ and $A_{1}$ are positive constants. In Fig. 4 we use a function that interpolates between these power-laws and allows us to fit $\langle z\rangle$ for the compression method over the entire range of $\alpha$. These arguments imply that $\langle z\rangle=\langle z\rangle_{\text {iso }}$ in the $\alpha \rightarrow \infty$ limit, yet this is still an open question. Previous studies have predicted that $\langle z\rangle(\alpha)-\langle z\rangle(1)$ scale as $\sqrt{\alpha-1}$ based on the behavior of the pair distribution function $g(r)$ near contact for spherical particle packings [9]. In contrast, our numerical results demonstrate hypostaticity in ellipse packings originates from the existence of quartic modes.

Annealed packings The ellipse packings discussed up to this point were generated using the compression method at fixed aspect ratio. Using this method, we obtained similar $\langle z\rangle(\alpha)$ to that obtained previously for hard ellipse packings [9]. Since ellipse packings are hypostatic, it is in principle possible to obtain packings with higher $\langle z\rangle$ than found in Fig. 4 without increasing the translational or orientational order. To test this, we developed an 'annealing' method, which creates static packings by incrementally increasing the aspect ratio from $\alpha=1$. We initially create bidisperse disk packings. Each disk is then assigned the same aspect ratio $1+\Delta \alpha$ with the direction of the long axis chosen randomly. A new ellipse packing is formed from this initial state using the compression method described above. The ellipses of the new packing are elongated again along their defined major axes, and the protocol is repeated until a packing with the desired aspect ratio is reached. Using this method, ellipse packings can be generated that are denser and possess contact numbers much closer to $z_{\text {iso }}=6$ as shown in Fig. 4. The annealed packings still exhibit a low-frequency gap, and the location, $i^{*}$, of this gap can be used to predict $\langle z\rangle$ through Eq. 4. The variation of $i^{*}$ with aspect ratio, however, differs from the 'compressed' packings. The annealed packings tend to have higher $\langle z\rangle$, fewer quartic modes, and a plateau in $\langle z\rangle$ at large $\alpha$.

In conclusion, we find that aspherical grains qualitatively change the nature of jamming at point J. Model systems with spherical grains appear to be the exceptional case: they are isostatic, and all nontrivial modes of excitation increase quadratically with deformation amplitude. In contrast, ellipse packings, which are more relevant for real granular media, possess quartic modes characterized by collective rotational motions. Thus, Landau theories for ellipse packings have a 4th order term as the lowest nonvanishing contribution to the generalized free energy. These results will likely stimulate further work on statistical descriptions of packings of aspherical particles.

Support from NSF grant numbers DMR-0549762 (BC \& MM), DMR-0448838 (CSO) and CDI-0835742 (CS) is acknowledged. During the workshop "Dynamical heterogeneities in glasses, colloids, and granular media" in Leiden in August 2008, we learned of independent, parallel results by Zeravcic, et al. [23] on 3D ellipsoids, with similar conclusions. We acknowledge discussions and suggestions from $\mathrm{Z}$. Zeravcic and S. Nagel at the workshop, and also thank M. Bi, G. Lois, T. Witten, and N. Xu for helpful comments.

[1] A. J. Liu and S. R. Nagel, Nature 396, 21 (1998).

[2] C. S. O’Hern, L. E. Silbert, A. J. Liu, and S. R. Nagel, Phys. Rev. E 68, 011306 (2003).

[3] H. A. Makse, D. L. Johnson, and L. M. Schwartz, Phys. Rev. Lett. 84, 4160 (2000).

[4] C. S. O'Hern, S. A. Langer, A. J. Liu, and S. R. Nagel, Phys. Rev. Lett. 88, 075507 (2002).

[5] M. Wyart, S. R. Nagel, and T. A. Witten, Europhys. Lett. 72, 486 (2005).

[6] M. Wyart, L. E. Silbert, S. R. Nagel, and T. A. Witten, Phys. Rev. E 72, 051306 (2005).

[7] E. Somfai, M. Van Hecke, W. G. Ellenbroek, K. Shundyak, and W. Van Saarloos, Phys. Rev. E 75, 020301(R) (2007).

[8] C. F. Moukarzel, Phys. Rev. Lett. 81, 1634 (1998); R. Blumenfeld, Phys. Rev. Lett. 93, 108301 (2004).

[9] A. Donev, R. Connelly, F. H. Stillinger, and S. Torquato, Phys. Rev. E 75, 051304 (2007).

[10] A. Donev, I Cisse, D. Sachs, E. A. Variano, F. H. Stillinger, R. Connelly, and S. Torquato, Science 303, 990 (2004).

[11] G.-J. Gao, J. Blawzdziewicz, and C. S. O'Hern, Phys. Rev. E 74, 061304 (2006).

[12] H. P. Zhang and H. A. Makse, Phys. Rev. E 72, 011301 (2005).

[13] J.W. Perram and M.S. Wertheim, J. Comp. Phys. 58, 409 (1985).

[14] J.W. Perram, J. Rasmussen, E. Praestgaard, J.L. Lebowitz, Phys. Rev. E 54, 6565 (1996).

[15] B. J. Berne and P. Pechukas, J. Chem. Phys. 46, 4213 (1972).

[16] D. J. Cleaver, C. M. Care, M. P. Allen, and M. P. Neal, Phys. Rev. E 54, 559 (1996).

[17] The parameters $\sigma_{0}, \beta$, and $\chi$ in Eq. 2 are defined as $\sigma_{0}=\sqrt{\frac{b_{i}^{2}}{\lambda}+\frac{b_{j}^{2}}{1-\lambda}}, \quad \beta=\left(\frac{\left(a_{i}^{2}-b_{i}^{2}\right)\left(a_{j}^{2}+\frac{1-\lambda}{\lambda} b_{i}^{2}\right)}{\left(a_{j}^{2}-b_{j}^{2}\right)\left(a_{i}^{2}+\frac{\lambda}{1-\lambda} b_{j}^{2}\right)}\right)^{1 / 4}$, and $\chi=$ $\left(\frac{\left(a_{i}^{2}-b_{i}^{2}\right)\left(a_{j}^{2}-b_{j}^{2}\right)}{\left(a_{j}^{2}+\frac{1-\lambda}{\lambda} b_{i}^{2}\right)\left(a_{i}^{2}+\frac{\lambda}{1-\lambda} b_{j}^{2}\right)}\right)^{1 / 2}$.

[18] J. A. Cuesta and D. Frenkel, Phys. Rev. A 42, 2126 (1990).

[19] A. Tanguy, J. P. Wittmer, F. Leonforte, and J.-L. Barrat, Phys. Rev. B 66, 174205 (2002).

[20] In the following discussion, we have accounted for rattler ellipses and set $N=N^{\prime}$.

[21] M. Mailman, C. Schreck, B. Chakraborty, and C. S. O’Hern (unpublished).

[22] J. G. Gay and B. J. Berne, J. Chem. Phys. 74, 3316 (1981).

[23] Z. Zeravcic, N. Xu, et al. (unpublished). 